\title{
Ultrafast nuclear dynamics in the doubly-core-ionized water molecule observed via Auger spectroscopy
}

\author{
T. Marchenko, ${ }^{1,2,{ }^{*}}$ L. Inhester,,${ }^{3,4, \dagger}$ G. Goldsztejn, ${ }^{1}$ O. Travnikova,,${ }^{1,2}$ L. Journel,,${ }^{1,2}$ R. Guillemin, ${ }^{1,2}$ I. Ismail, ${ }^{1}$ \\ D. Koulentianos, ${ }^{5,1}$ D. Céolin, ${ }^{2}$ R. Püttner, ${ }^{6}$ M. N. Piancastelli, ${ }^{1,7}$ and M. Simon ${ }^{1,2}$ \\ ${ }^{1}$ Sorbonne Université, CNRS, Laboratoire de Chimie Physique-Matière et Rayonnement, LCPMR, F-75005, Paris, France \\ ${ }^{2}$ Synchrotron SOLEIL, l'Orme des Merisiers, Saint-Aubin, BP 48, F-91192 Gif-sur-Yvette Cedex, France \\ ${ }^{3}$ Center for Free-Electron Laser Science, DESY, Notkestrasse 85, 22607 Hamburg, Germany \\ ${ }^{4}$ The Hamburg Centre for Ultrafast Imaging, Luruper Chaussee 149, D-22761 Hamburg, Germany \\ ${ }^{5}$ Department of Physics, University of Gothenburg, Origovägen 6B, SE-412 96 Gothenburg, Sweden \\ ${ }^{6}$ Fachbereich Physik, Freie Universität Berlin, Arnimallee 14, D-14195 Berlin, Germany \\ ${ }^{7}$ Department of Physics and Astronomy, Uppsala University, PO Box 516, SE-751 20 Uppsala, Sweden
}

(Received 14 June 2018; published 3 December 2018)

\begin{abstract}
We present a combined experimental and theoretical study of the Auger-emission spectrum following double core ionization and excitation of gas-phase water molecules with hard-x-ray synchrotron radiation above the $\mathrm{O} \mathrm{K}^{-2}$ threshold. We observe an indication of ultrafast proton motion occurring within the $1.5 \mathrm{fs}$ lifetime of the double-core-hole $(\mathrm{DCH})$ states in water. Furthermore, we have identified symmetric and antisymmetric dissociation modes characteristic for particular DCH states. Our results serve as a fundamental reference for state-of-the-art studies of DCH dynamic processes in liquid water both at synchrotron and free-electron-laser facilities.
\end{abstract}

DOI: 10.1103/PhysRevA.98.063403

\section{INTRODUCTION}

Considered as the most important liquid in nature, water remains the subject of unceasing interest for natural scientists due to its key role in chemistry and biology, since most chemical reactions and biological functions take place in aqueous environments, as well as due to its anomalous properties [1]. Various $x$-ray spectroscopy techniques, sensitive to the chemical environment of a specific element, proved to be an efficient probe of the electronic and hydrogen-bonding structure of liquid water [2]. At the same time, in a living cell $\mathrm{x}$-ray ionization of water may lead to a significant indirect radiation damage caused by the $\mathrm{OH}$ radicals and electrons formed through a cascade of reactions and secondary ionization processes [3]. Therefore, knowledge of the dynamic response of water to the ionizing $\mathrm{x}$-ray radiation is essential.

Multiple examples of experimental and theoretical studies on electron and nuclear dynamics in aqueous solutions in the soft-x-ray domain are discussed in Refs. [4-8] and references therein. Recently, electron transfer processes from solvent water molecules to the solvated cation in the hard-x-ray regime have been observed [9]. However, the simultaneous presence of electron and nuclear dynamics in x-ray-excited liquid water hinders the interpretation of the measurements. Namely, the nuclear dynamics of hydrogen atoms, occurring in water excited at the oxygen $K$ edge, may influence the effect of electron delocalization and solvation through the hydrogen-bonding network [10].

\footnotetext{
*tatiana.marchenko@upmc.fr

†ludger.inhester@cfel.de
}

One of the possibilities to reduce the effect of nuclear dynamics, proposed in Ref. [11], refers to double-core-hole (DCH) spectroscopy. Since the lifetimes of the DCH states can be significantly shorter than the lifetimes of the corresponding single-core-hole states (SCHs) [12-15], one can expect a considerable reduction of the nuclear dynamics in $\mathrm{DCH}$ states. However, as we have recently shown, strongly dissociative potential-energy surfaces of molecular DCH states may provide for a substantial nuclear dynamics [15] and may even lead to an ultrafast dissociation [16]. Our earlier theoretical investigations of gas-phase water molecules predicted fast dissociative dynamics in the oxygen $K^{-2}$ states, which should be observable in the Auger spectra of these states [17].

In this paper we present a combined experimental and theoretical study of the Auger-emission spectrum following double core ionization and excitation of gas-phase water molecules with hard-x-ray synchrotron radiation above the $\mathrm{O}$ $K^{-2}$ threshold. We observe an indication of ultrafast proton motion occurring within the $1.5 \mathrm{fs}$ lifetime of the DCH states in water. Furthermore, we have identified symmetric and antisymmetric dissociation modes characteristic for particular DCH states. Our results serve as a fundamental reference for state-of-the-art studies on DCH dynamic processes in liquid water both at synchrotron and free-electron-laser facilities.

\section{EXPERIMENT}

The experiment has been performed at the HAXPES end station, based on a hemispherical electron analyzer installed on the GALAXIES beam line at the synchrotron SOLEIL $[18,19]$. The spectrometer is set parallel to the light polarization vector. The photon energy of $2.3 \mathrm{keV}$ significantly 


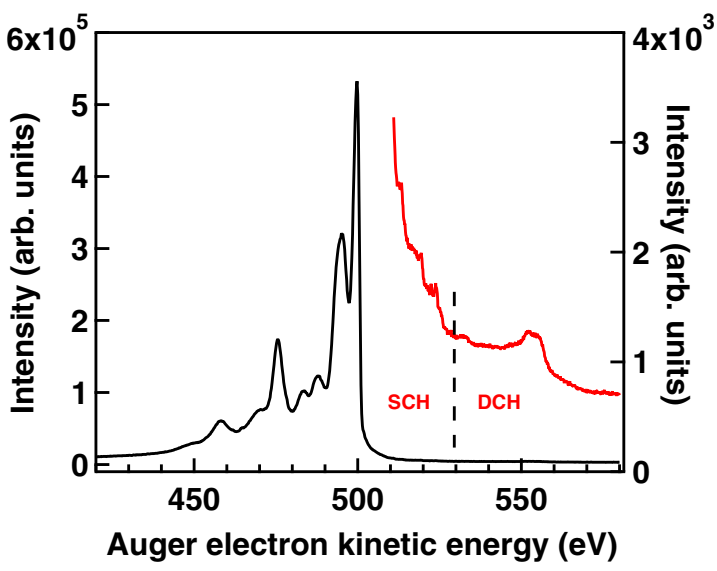

FIG. 1. Oxygen $K L L$ Auger spectrum in water at $2.3 \mathrm{keV}$ photon energy (black, left axis) and an enlarged view of the satellite and hypersatellite Auger lines recorded with higher statistics (red, right axis).

exceeds the oxygen $K^{-2}$ double-photoionization threshold located at $1171 \mathrm{eV}$ [20]. As we have recently shown in neon [21], single-photon hard-x-ray ionization leads to formation of various types of DCH states: $K^{-2}$ with two core electrons emitted to the continuum, $K^{-2} V$ with one core electron in the continuum and the other one in an unoccupied valence orbital $V$, and $K^{-2} L^{-1}$ and $K^{-2} L^{-1} V$ states, where the formation of a DCH state is accompanied by a shake process of an electron from an occupied valence orbital $L$. The transitions from such intermediate DCH states form the hypersatellite (HS) Auger decay spectrum. Figure 1 shows the oxygen $K L L$ Auger spectrum, recorded with a total experimental resolution of $0.6 \mathrm{eV}$, which includes the spectrometer resolution and the thermal Doppler broadening. The dominant lines in the spectrum at the kinetic energy of $500 \mathrm{eV}$ and below reproduce the previously reported results obtained in gas-phase water by electron impact [22]. However, the enlarged view of the highenergy tail of the spectrum, measured with higher statistics, shows a rich structure formed by the satellite and the HS Auger lines. The satellites at the kinetic energy between 500 and $530 \mathrm{eV}$ correspond to the Auger transitions from the $\mathrm{O} K^{-1} L^{-1} V$ states resulting from single $\mathrm{O} K^{-1}$ ionization accompanied by shake-up excitation of an electron from an occupied valence shell $L$ to an unoccupied valence shell $V$. These peaks can be equally observed in the soft-x-ray regime below the $K^{-2}$ double-photoionization threshold [23]. The HS Auger lines, originating from the decay of the DCH states, are located at kinetic energies above $530 \mathrm{eV}$. For further analysis, we zoom in on the 530-580 eV kinetic-energy range and subtract the Lorentzian tail of the dominant $K L L$ Auger line in order to isolate the much weaker (factor of $10^{3}$ ) HS features. The resulting experimental DCH HS Auger spectrum is shown in Fig. 2.

\section{THEORY}

To extract information on the dynamics in the DCH states of water, we have performed calculations of the HS Auger spectra by using the formalism outlined in Ref. [17]. The

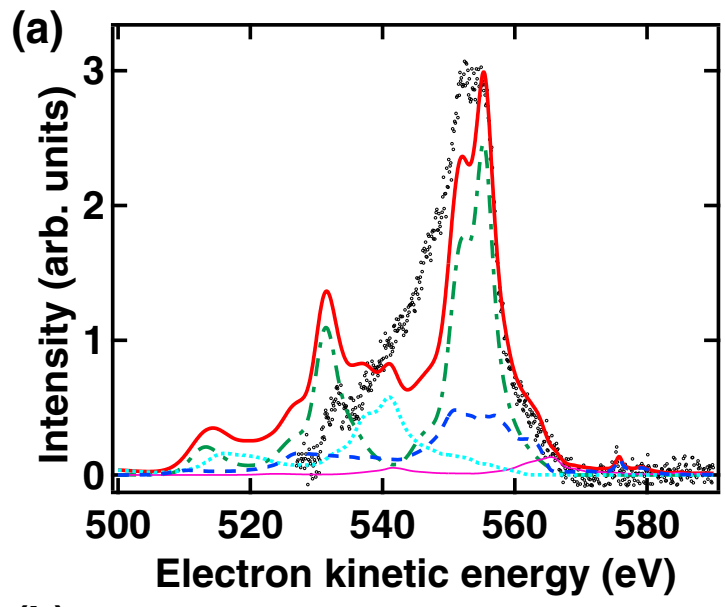

(b)

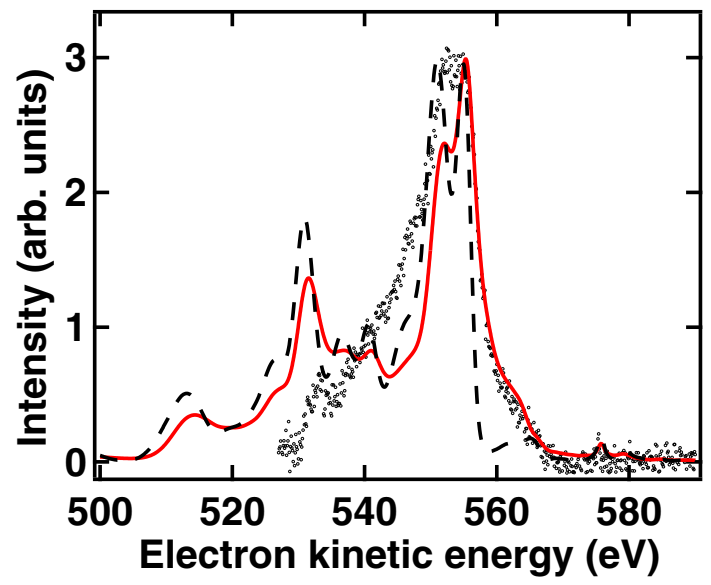

FIG. 2. (a) Comparison of an experimental hypersatellite Auger spectrum of water measured at $2.3 \mathrm{keV}$ (black circles) with the Auger spectra calculated including the nuclear dynamics. The total Auger spectrum (thick solid red line) is composed of various hypersatellite contributions: $K^{-2}$ (dash-dotted green), $K^{-2} V$ (thin solid magenta), $K^{-2} L^{-1}$ (dotted cyan), and $K^{-2} L^{-1} V$ (dashed blue) states. (b) Comparison of the experimental HS Auger spectra (black circles) to the calculation with fixed molecular geometry (dashed black) and with the nuclear dynamics included (solid red).

Auger lineshape $T(E)$ for the Auger electron stemming from the decay of an electronic core hole state $c$, which has been populated by the ionization of a neutral molecule in its ground state, to a final electronic state $f$ is given by the KramersHeisenberg formula,

$$
T(E)=\frac{\Gamma_{c \rightarrow f}}{2 \pi} \sum_{n_{f}}\left|\sum_{n_{c}} \frac{\left\langle n_{f} \mid n_{c}\right\rangle\left\langle n_{c} \mid 0\right\rangle}{E-\left(E_{n_{c}}-E_{n_{f}}\right)+i \Gamma / 2}\right|^{2},
$$

where $\left|n_{c}\right\rangle$ and $\left|n_{f}\right\rangle$ denote vibrational eigenstates of the intermediate, core ionized, and final electronic state with energy $E_{n_{c}}$ and $E_{n_{f}}$, respectively. The ket vector $|0\rangle$ is the initial ground state and $\Gamma=\sum_{f} \Gamma_{c \rightarrow f}$ is the total decay width of the core hole state. In Eq. (1) it is assumed that the Auger transition rate for going from electronic state $c$ to electronic state $f$ by emitting an Auger electron, $\Gamma_{c \rightarrow f}$, is independent of variations of the molecular geometry. 
Equation (1) can be rewritten with integration over time as [24]

$$
\begin{aligned}
T(E)= & \frac{\Gamma_{c \rightarrow f}}{2 \pi} \int_{0}^{\infty} d t e^{-\Gamma t} \\
& \times \int d^{3} q^{\prime} \int d^{3} q\left\langle 0\left|e^{i H_{c} t}\right| \mathbf{q}^{\prime}\right\rangle\left\langle\mathbf{q}\left|e^{-i H_{c} t}\right| 0\right\rangle \\
& \times \int_{-t}^{t} d \tau e^{i E \tau}\left\langle\mathbf{q}^{\prime}\left|e^{-i H_{c} \tau / 2} e^{i H_{f} \tau} e^{-i H_{c} \tau / 2}\right| \mathbf{q}\right\rangle,
\end{aligned}
$$

where the integrals over $\mathbf{q}$ and $\mathbf{q}^{\prime}$ are over the spatial coordinates of the molecule and we have exploited the fact that $\left|n_{c}\right\rangle$ and $\left|n_{f}\right\rangle$ are eigenstates of the Hamilton operators $H_{c}$, $H_{f}$ that describe the dynamics in the core-ionized state and the final electronic state, respectively.

The $\tau$ integral in Eq. (2) dominantly contributes for small times $|t|<1 / \Gamma$. Using the Baker-Campbell-Hausdorff formula [25] we approximate the integrand as

$$
\begin{gathered}
e^{i E \tau}\left\langle\mathbf{q}^{\prime}\left|e^{-i H_{c} \tau / 2} e^{i H_{f} \tau} e^{-i H_{c} \tau / 2}\right| \mathbf{q}\right\rangle \\
=\left\langle\mathbf{q}^{\prime}\left|e^{i\left[E-\left(H_{c}-H_{f}\right)\right] \tau+O\left(\tau^{3}\right)}\right| \mathbf{q}\right\rangle \\
\simeq e^{i\left\{E-\left[V_{c}(\mathbf{q})-V_{f}(\mathbf{q})\right]\right\} \tau} \delta\left(\mathbf{q}-\mathbf{q}^{\prime}\right) .
\end{gathered}
$$

In Eq. (3) all the second-order terms $O\left(\tau^{2}\right)$ in the exponent cancel out, such that only $O\left(\tau^{3}\right)$ terms remain that involve nested commutators of $H_{c}$ and $H_{f}$.

The above approximation allows us to write the Auger lineshape as

$$
\begin{aligned}
T(E)= & \frac{\Gamma_{c \rightarrow f}}{2 \pi} \int_{0}^{\infty} d t e^{-\Gamma t} \int d^{3} q\left|\left\langle 0\left|e^{i H_{c} t}\right| \mathbf{q}\right\rangle\right|^{2} \\
& \times \frac{2 \sin \left\{\left[\Delta V_{c \rightarrow f}(\mathbf{q})-E\right] 2 t\right\}}{\Delta V_{c \rightarrow f}(\mathbf{q})-E},
\end{aligned}
$$

where $\Delta V_{c \rightarrow f}(\mathbf{q})=V_{c}(\mathbf{q})-V_{f}(\mathbf{q})$ is the difference of the potential-energy surfaces of the core ionized and final electronic states at molecular geometry $\mathbf{q}$.

The integrand in Eq. (5) contains the weighting factor $e^{-\Gamma t}$ describing the decay of the core-hole state and the squared density of the wave packet propagated on the potential-energy surface of the core-ionized state, $\left|\left\langle 0\left|e^{i H_{c} t}\right| \mathbf{q}\right\rangle\right|^{2}$. When considering a static wave packet, $\left|\left\langle\mathbf{q}\left|e^{-i H_{c} t}\right| 0\right\rangle\right|^{2} \simeq|\langle\mathbf{q} \mid 0\rangle|^{2}$, and the time integration yields

$$
T(E)=\frac{\Gamma_{c \rightarrow f}}{2 \pi} \int d^{3} q \frac{|\langle 0 \mid \mathbf{q}\rangle|^{2}}{\left[\Delta V_{c \rightarrow f}(\mathbf{q})-E\right]^{2}+(\Gamma / 2)^{2}},
$$

which is a Lorentzian describing the finite lifetime of the corehole state convoluted with the static-wave-packet distribution.

Instead of a static wave packet, we assume a dynamical wave packet for which the energy separation $\Delta V_{c \rightarrow f}(\mathbf{q})$ slowly evolves compared with the timescales of the decay $\tau=1 / \Gamma$. Accordingly, we approximate the term

$$
\frac{2 \sin \{[\Delta V(\mathbf{q})-E] 2 t\}}{\Delta V(\mathbf{q})-E}
$$

in the integration kernel with an effective Lorentzian with a full width at half maximum FWHM $=1 / \Gamma$,

$$
T(E) \simeq \int d t e^{-\Gamma t} T(E, t)
$$

with

$$
T(E, t)=\frac{\Gamma_{c \rightarrow f}}{2 \pi} \int d^{3} q \frac{\left|\left\langle\mathbf{q}\left|e^{-i H_{c} t}\right| 0\right\rangle\right|^{2}}{\left(\Delta V_{c \rightarrow f}(\mathbf{q})-E\right)^{2}+(\Gamma / 2)^{2}} .
$$

In Eq. (7) the lineshape can be read as an incoherent superposition of instantaneous spectra $T(E, t)$ at time $t$ weighted with the factor $\exp (-\Gamma t)$ describing the decay of the corehole-state population. The dominant broadening in the spectra is due to the vibrational width via convolution with the wave packet. The width due to finite lifetime is only a relatively small part (for the DCH state in water the lifetime corresponds to a FWHM of $0.44 \mathrm{eV}$ and the vibrational broadening contributes on top of that with a FWHM of $2.2 \mathrm{eV}$ ). We therefore further simplify this expression and use instead of the Lorentzian the corresponding Gaussian distribution,

$$
T(E, t) \simeq \frac{\Gamma_{c \rightarrow f}}{\sqrt{2 \pi} \sigma_{\text {lifetime }} \Gamma} \int d^{3} q\left|\left\langle\mathbf{q}\left|e^{-i H_{c} t}\right| 0\right\rangle\right|^{2} e^{\frac{-\left[\Delta V_{c \rightarrow f}(\mathbf{q})-E\right]^{2}}{2 \sigma_{\text {lifetime }}^{2}}}
$$

with $\sigma_{\text {lifetime }}=\frac{\Gamma}{2 \sqrt{2 \ln 2}}$.

To evaluate the spectral lineshape, we assume that the wave packet maintains its Gaussian shape during the lifetime of the core-hole state $\tau=1 / \Gamma$. The center of the wave packet, as well as its width, changes in time due to dissociation. Expanding $\Delta V_{c \rightarrow f}(\mathbf{q})$ up to the first-order derivative and taking into account additional experimental broadening $\sigma_{\text {exp }}$, the instantaneous lineshape becomes

$$
T(E, t)=\frac{\Gamma_{c \rightarrow f}}{\Gamma \sqrt{2 \pi} \sigma_{E}(t)} \exp \left\{\frac{-\left[\Delta V_{c \rightarrow f}\left(\mathbf{q}_{c}(t)\right)-E\right]^{2}}{2 \sigma_{E}^{2}(t)}\right\}
$$

with the instantaneous $\Delta V_{c \rightarrow f}\left(\mathbf{q}_{c}(t)\right)$ calculated by using the time-dependent center position of the vibrational wave packet $\mathbf{q}_{c}(t)$ and

$$
\sigma_{E}(t)^{2}=\sum_{i}\left[\frac{d \Delta V_{c \rightarrow f}\left(\mathbf{q}_{c}(t)\right)}{d q_{i}} \sigma_{i}(t)\right]^{2}+\sigma_{\exp }^{2}+\sigma_{\text {lifetime }}^{2}
$$

In Eq. (11) the sum runs over the vibrational modes in the water molecule, $\frac{d \Delta V_{c \rightarrow f}\left(\mathbf{q}_{c}(t)\right)}{d q_{i}}$ is the derivative of the potential-energy surface difference along the vibrational mode coordinate $q_{i}$ at the center of the wave packet at time $t$, and $\sigma_{i}(t)$ is the time-dependent width (standard deviation) of the wave packet along the normal coordinate at time $t$.

Because the derivative along the antisymmetric mode is zero by symmetry, the broadening due to antisymmetric stretching is not yet taken into account via Eq. (11). To also implement the effects of dissociation along the antisymmetric stretching mode, we have expanded the potentialenergy-surface difference up to the second derivative along the antisymmetric stretch mode, $d^{2} \Delta V_{c \rightarrow f}\left(\mathbf{q}_{c}(t)\right) / d q_{a}^{2}$. With the effects of antisymmetric dissociation included, the 
instantaneous lineshape reads

$$
T(E, t)=\int d q_{a} p_{a}\left(q_{a}, t\right) \frac{1}{\sqrt{2 \pi} \sigma_{E}} \exp \left\{\frac{-\left[\Delta V_{c \rightarrow f}\left(\mathbf{q}_{c}(t)\right)+\frac{1}{2} \frac{d^{2} \Delta V_{c \rightarrow f}\left(\mathbf{q}_{c}(t)\right)}{d q_{a}^{2}} q_{a}^{2}-E\right]^{2}}{2 \sigma_{E}^{2}}\right\},
$$

where $\sigma_{E}$ is the width according to Eq. (11) for the symmetric stretching and bending mode, and the integral is performed along the antisymmetric stretching mode coordinate $q_{a}$, for which we employ the spatial distribution

$$
p_{a}\left(q_{a}, t\right)=\frac{1}{\sqrt{2 \pi} \sigma_{a}(t)} \exp \left(-\frac{q_{a}^{2}}{2 \sigma_{a}^{2}(t)}\right),
$$

where $\sigma_{a}(t)$ is the spatial width of the wave packet along the antisymmetric stretching mode at time $t$. Note that this integral causes an asymmetric broadening: for $d^{2} \Delta V_{c \rightarrow f} / d q_{a}^{2}>$ 0 the line is shifted to lower energies, for $d^{2} \Delta V_{c \rightarrow f} / d q_{a}^{2}<0$ the line is shifted to higher energies. Using molecular dynamics simulations, we followed the propagation of the wave packet along the potential-energy surfaces of the core-excited states and obtained the time-dependent nuclear wave packet $\rho(\mathbf{q}, t)$.

Auger decay rates $\Gamma_{c \rightarrow f}$ were calculated by using the procedure described in Ref. [17]. In essence, initial and final electronic states are calculated by using a configuration interaction calculation employing an orbital basis consisting of molecular orbitals optimized for the DCH state. To determine the wave function of the Auger electron, we conduct a singlecenter expansion of the electronic potential in the final Auger channel and solve it for the wave functions in the electronic continuum. For a better comparison with experiment the total spectrum is shifted by $2 \mathrm{eV}$ to lower energies. This small overall shift may be attributed to missing contributions in the electronic-structure calculation, such as relativistic contributions, correlation effects, and finite-basis-set effects.

\section{RESULTS}

In Fig. 2(a) the experimental DCH HS Auger spectrum obtained after the background subtraction is compared with the calculated Auger spectrum. Apart from the dominant DCH $K^{-2}$ state, the calculations include the contributions from $K^{-2} V, K^{-2} L^{-1}$, and $K^{-2} L^{-1} V$ states. For atoms it has been shown that the probability of different shake-off processes is mainly determined by the occupation of the corresponding subshells [26]. We therefore think that the relative weight for the different hypersatellites states is, to a good approximation, the same as for the isoelectronic neon atom [27]. According to our earlier measurements in neon, where HS contributions were identified with the electronic structure calculations as well as by their widths and shapes [21], the relative weights of different HS states were fixed here as $K^{-2}$ : $K^{-2} V:\left(K^{-2} L^{-1}+K^{-2} L^{-1} V\right)=0.56: 0.04: 0.4$. The relatively low contribution of $K^{-2} V$ hypersatellites is consistent with observations reported for the $\mathrm{N}_{2}$ molecule at high photon energies [28]. The relative weights of different $K^{-2} L^{-1}$ and $K^{-2} L^{-1} V$ states were left as free fitting parameters. The results of the fitting show that $K^{-2} L^{-1}$ and $K^{-2} L^{-1} V$ states provide nearly equal contributions to the total HS Auger spectrum. The obtained relative weights of different intermediate states are summarized in Table I, column A.

One can observe an overall agreement between experiment and the total calculated HS Auger spectrum. The dominant $K^{-2}$ contribution has a strong peak around $553 \mathrm{eV}$ and a peak around $531 \mathrm{eV}$, in agreement with the measurements. A small feature seen at $578 \mathrm{eV}$ can be associated with Auger decay of $K^{-2} L^{-1} V$ states.

Remarkably, a very good agreement is observed at the higher-energy part of the spectrum around $560 \mathrm{eV}$, where a marked tail was earlier predicted as a fingerprint of the nuclear dynamics in the doubly-core-ionized water molecule [17]. This tail, clearly observed in the experimental spectrum, appears in the calculations due to the dynamical broadening of all the HS peaks contributing in this energy region. The role of the nuclear dynamics is further revealed through comparison to the calculations with fixed molecular geometry, where fitting the relative weights of different HS contributions fails to reproduce the tail at $560 \mathrm{eV}$. As an illustration, Fig. 2(b) shows the shape of the total calculated Auger spectrum in the absence of the nuclear dynamics in comparison with the experiment and with the calculations including nuclear dynamics.

To eliminate other possible interpretations of the tail observed at $560 \mathrm{eV}$ we evaluate in the following the effects of several assumptions made in our work.

\section{A. Effect of line shape}

In our theoretical approach, we approximate in Eq. (9) the Lorentzian due to finite lifetime by the corresponding Gaussian function. Since a Lorentzian has a slower decaying

TABLE I. The relative weights of different intermediate electronic states contributing to the HS Auger spectrum. Column A shows weights that are according to the distribution found in neon; Column B shows weights with a reduced contribution for the main satellite $K^{-2}$ and a stronger contribution for the $K^{-2} L^{-1} V$ satellites.

\begin{tabular}{lccc}
\hline \hline Configuration & $\begin{array}{c}\text { Electronic } \\
\text { state }\end{array}$ & $\begin{array}{c}\text { Relative } \\
\text { weight (A) }\end{array}$ & $\begin{array}{c}\text { Relative } \\
\text { weight (B) }\end{array}$ \\
\hline$K^{-2}$ & $K^{-2}$ & 0.56 & 0.48 \\
$K^{-2} V$ & $K^{-2} 4 a_{1}$ & 0.02 & 0.05 \\
$K^{-2} V$ & $K^{-2} 2 b_{2}$ & 0.02 & 0.05 \\
$K^{-2} L^{-1} V$ & $K^{-2} 3 a_{1}^{-1} 4 a_{1}$ & 0.06 & 0.10 \\
$K^{-2} L^{-1} V$ & $K^{-2} 2 a_{1}^{-1} 4 a_{1}$ & 0.09 & 0.10 \\
$K^{-2} L^{-1} V$ & $K^{-2} 1 b_{2}^{-1} 2 b_{2}$ & 0.07 & 0.11 \\
$K^{-2} L^{-1}$ & $K^{-2} 3 a_{1}^{-1}$ & 0.03 & 0.02 \\
$K^{-2} L^{-1}$ & $K^{-2} 1 b_{2}^{-1}$ & 0.07 & 0.08 \\
$K^{-2} L^{-1}$ & $K^{-2} 2 a_{1}^{-1}$ & 0.09 & 0.02 \\
\hline \hline
\end{tabular}




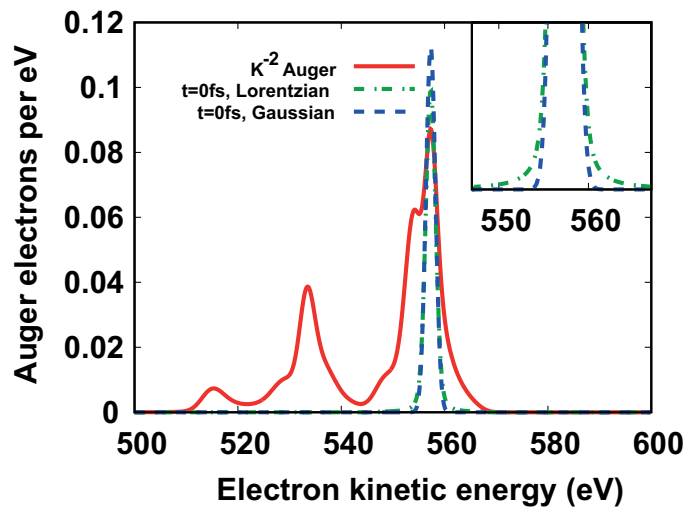

FIG. 3. Comparison of calculated Auger line profile for one of the dominant Auger channels $\left(K^{0} \rightarrow K^{1} 1 b_{1}^{-2}\right)$ employing the Gaussian approximation in Eq. (9) and without. The solid red line shows the full calculated Auger spectrum, the dash-dotted green and dashed blue lines show the instantaneous ( $t=0 \mathrm{fs}$ ) Auger line profile for a single Auger channel. For the dash-dotted green line, the broadening due to finite lifetime was incorporated through a Lorentzian, and for the dashed blue line by employing a corresponding Gaussian. The inset shows a magnified region around the tail of the peak.

tail, the long tail seen in the experimental Auger spectrum might also arise to a certain degree from the Lorentzian tail. To verify our approximation we have calculated the Auger line profile for a single Auger transition at time $t=0$ fs by using a Lorentzian instead of a Gaussian lifetime broadening. As can be seen in Fig. 3, the effects on the lineshape are hardly visible, and therefore cannot be be responsible for the appearance of the tail extended to higher energies discussed in Fig. 2.

\section{B. Effect of relative weights}

Due to the more extended spatial geometry of the electronic structure in water compared with neon, the creation of higher hypersatellites might be facilitated in water compared with neon. One might expect that the relative weight of the $K^{-2} L^{-1} V$ and $K^{-2} V$ satellites might thus be a bit higher than for neon. To account for this uncertainty, we also conducted a fit where we have assumed a lower weight for the $K^{-2}$ main satellite. The relative weights of the contributing electronic states, obtained as a result of this alternative fitting procedure, are summarized in Table I, column B. The resulting spectra with and without nuclear dynamics are shown in Fig. 4. The vertical lines in Fig. 4(b) show the Auger transitions of the dominant $K^{-2}$ main satellite line. As can be seen, without inclusion of nuclear dynamics the transition involving the fastest Auger electrons $\left(1 b_{1}^{-2}\right.$ channel) is below $558 \mathrm{eV}$. Even a stronger contribution of satellite weights $K^{-2} L^{-1} V$ cannot explain the observed tail above $558 \mathrm{eV}$.

\section{Effect of postcollision interaction}

For the $K^{-2}$ double-core-hole hypersatellites two photoelectrons are produced. The excess energy of the photoionization process is split up into kinetic energies of these two photoelectrons in a U-shaped distribution. Typically a fast photoelectron carries away most of the excess energy and another slow photoelectron is emitted with kinetic energy lower than those of the subsequent Auger electrons. The energy of the Auger electron and its lineshape is altered when it overtakes this slower photoelectron. In case the Auger electron is much faster than the slow photoelectron, the instantaneous lineshape of the Auger electron due to this postcollision interaction (PCI) is $[29,30]$

$$
\begin{aligned}
T(E, t)= & \frac{\Gamma_{c \rightarrow f}}{2 \pi} \int d^{3} q\left|\left\langle 0\left|e^{i H_{c} t}\right| \mathbf{q}\right\rangle\right|^{2} \\
& \times \frac{\pi \xi}{\sinh (\pi \xi)} \frac{\exp \left[2 \xi \arctan \left(\frac{2\left[\Delta V_{c \rightarrow f}(\mathbf{q})-E\right]}{\Gamma}\right)\right]}{\left[\Delta V_{c \rightarrow f}(\mathbf{q})-E\right]^{2}+(\Gamma / 2)^{2}},
\end{aligned}
$$

with

$$
\xi=\frac{-Q_{d}}{v_{\mathrm{PE}}} \text { and } Q_{d}=1-\frac{v_{P E}}{v_{A E}},
$$

where $v_{\mathrm{PE}}$ and $v_{\mathrm{AE}}$ is the velocity in atomic units of the slow photoelectron and the Auger electron, respectively.

To estimate the effects of PCI on the shape of the HS Auger spectrum in water we have calculated the Auger line profile for a single Auger transition at time $t=0 \mathrm{fs}$
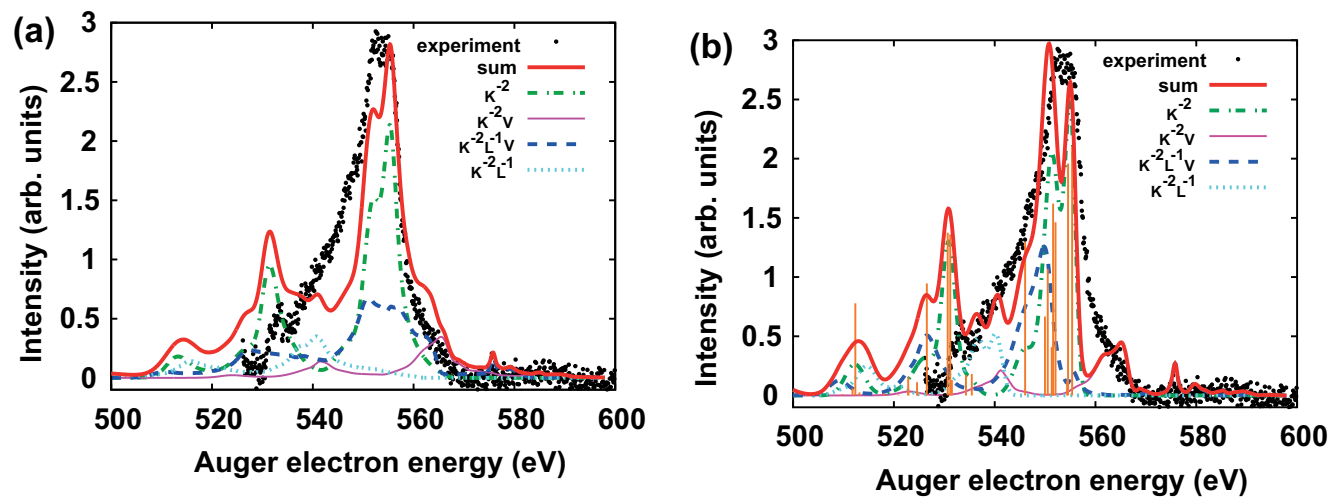

FIG. 4. Resulting Auger spectra (a) with and (b) without the effects of nuclear dynamics in the core-ionized state. The weights of the individual satellites are described in Table I, column B. (b) The vertical orange lines show the Auger transitions for the $K^{-2}$ satellite at the optimized ground-state geometry. 


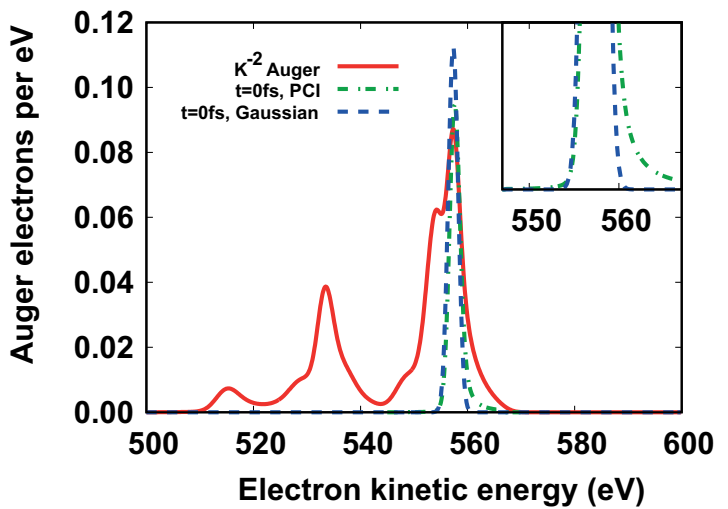

FIG. 5. Comparison of calculated Auger line profile for the dominant Auger channel $\left(1 b_{1}^{-2}\right)$ employing the Gaussian approximation in Eq. (9) (dashed blue line) and employing the PCI lineshape as given in Eq. (14) with $\xi=-0.46$ (dash-dotted green line). The solid red line shows the full calculated Auger spectrum, the green and blue lines show the instantaneous ( $t=0 \mathrm{fs}$ ) Auger line profile for a single Auger channel. The inset shows a magnified region around the tail of the peak.

assuming a parameter of $\xi=-0.46$ that was observed in the Auger-hypersatellite spectrum of neon [13]. This parameter corresponds to a slow photoelectron with kinetic energy of $\simeq 40 \mathrm{eV}$. Figure 5 compares the instantaneous spectra at $t=0$ fs for the Auger channel $K^{0} \rightarrow K^{1} 1 b_{1}^{-2}$. As can be seen, the PCI effect slightly shifts the peak position of the spectra to higher energies and leads to an asymmetric broadening that leave tails on the high-energy side of the peaks.

For the water molecule, the double-core-hole binding energy $(\simeq 1171 \mathrm{eV})$ [20]) is much lower than for neon ( $\simeq 1863 \mathrm{eV}$ [31]). Since for both experiments the same photon energy ( $2.3 \mathrm{keV})$ was used, the excess energy that the two photoelectrons share is much larger for water than for neon. We expect therefore that both photoelectrons that are produced along with the creation of double-core-hole hypersatellites in water are faster than those produced in the corresponding process in atomic neon. Since the PCI effect depends strongly on the velocity of the slow photoelectron, we expect the PCI effect to be weaker for the water molecule than for neon. Our comparison of the PCI effect in Fig. 5 represents therefore an upper limit of the lineshape distortion. An asymmetric broadening and a small shift to higher energies due to the PCI effect is insignificant for the dominant $K^{-2}$ line and, therefore, cannot be responsible for the tail observed at $560 \mathrm{eV}$.

However, the PCI effects may be significantly stronger for the $K^{-2} L^{-1}$ lines corresponding to emission of three photoelectrons due to the creation of additional valence vacancies. Among the three photoelectrons produced, we can expect two to have a velocity lower than the Auger electron. This effect cannot be accounted for in the calculations and may be responsible for the discrepancies observed between the calculated and the experimental spectrum at 540-550 eV.

\section{DISCUSSION}

The evolution of molecular geometry of a water molecule upon core ionization is an issue essential for applications.
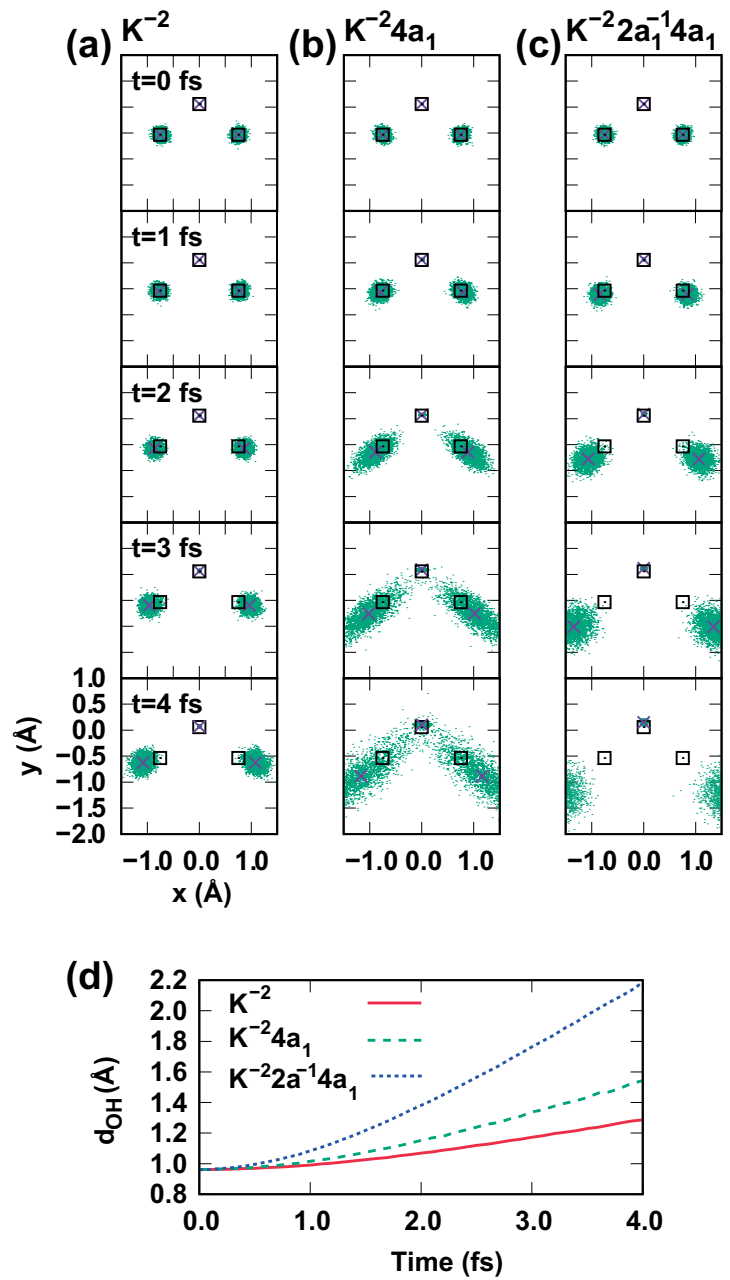

FIG. 6. Dynamics of the water molecule in the (a) $K^{-2}$, (b) $K^{-2} 4 a_{1}$, and (c) $K^{-2} 2 a_{1}^{-1} 4 a_{1}$ states. The crosses mark the peak of the Wigner distribution of the vibrational wave packet. The squares indicate the atom positions at $t=0 \mathrm{fs}$ in the neutral ground state optimized geometry. The green dots indicate the spatial Wigner distribution of the vibrational wave packet. (d) Evolution of the average $\mathrm{O}-\mathrm{H}$ bond distance as a function of time for the three electronic states.

Earlier theoretical studies demonstrated that, in the case of single-core-ionization $K^{-1}$, the bending mode is dominant, whereas dissociation is absent $[17,32]$. However, in the singlycore-excited $K^{-1} 4 a_{1}$ state, the antibonding character of the $4 a_{1}$ orbital was predicted to drive antisymmetric dissociation into $\mathrm{H}+\mathrm{OH}$ during the 4.1 fs lifetime of a single core hole $[33,34]$.

In the case of double core ionization $K^{-2}$, our calculations predict symmetric dissociation into $\mathrm{H}^{+}+\mathrm{O}+\mathrm{H}^{+}$within several femtoseconds [17]. The molecular dynamics in $K^{-2}$ state within the first few femtoseconds is shown in Fig. 6(a). In the figure the squares indicate the atom positions at $t=0 \mathrm{fs}$ in the neutral ground state optimized geometry and the green dots indicate the spatial Wigner distribution of the vibrational wave packet. One can see that the protons are expelled from the molecule in a symmetric way, while the spatial distribution of the vibrational wave packet remains essentially localized. 


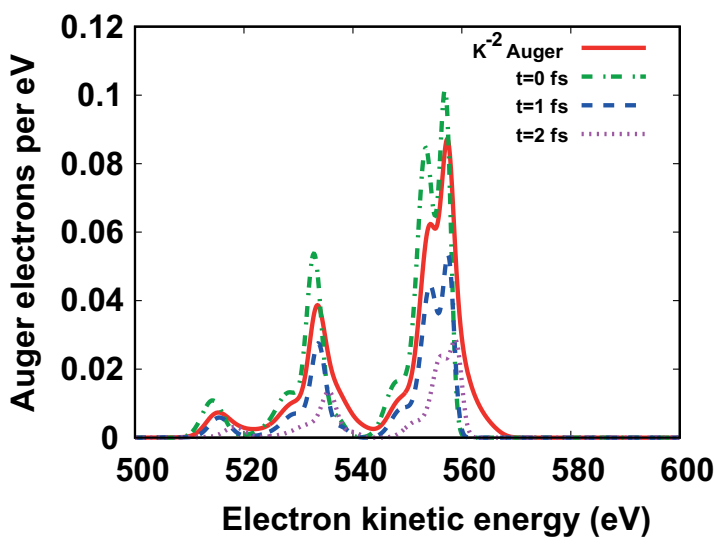

FIG. 7. Auger decay spectrum for the double $K$-shell ionized water molecule. The solid red line shows the assembled Auger spectrum according to Eq. (7). The dash-dotted/dashed/dotted lines show the instantaneous Auger spectra for different snapshots in time weighted by $\exp (-\Gamma t)$.

The effect of this fast dissociation on the Auger spectrum is demonstrated in Fig. 7, where instantaneous Auger spectra at different time steps are shown. As can be seen, with proceeding dissociation the Auger line of the dominant $1 b_{1}^{-2}$ channel shifts to higher energies, causing the marked tail towards higher energies in the Auger spectrum.

The molecular dynamics in the $K^{-2} 4 a_{1}$ state is shown in Fig. 6(b). Although the $K^{-2} 4 a_{1}$ state is only singly charged, the dissociative motion is faster in this case owing to the antibonding character of the $4 a_{1}$ orbital. In contrast to the $K^{-2}$ state, the nuclear dynamics in the $K^{-2} 4 a_{1}$ state has a strong contribution along the antisymmetric stretching mode, resulting in one short and one long bond distance. This leads to a much stronger smearing out of the spatial Wigner distribution. This finding corroborates an earlier theoretical prediction that both symmetric and antisymmetric stretching of the $\mathrm{O}-\mathrm{H}$ bond might be involved in the nuclear dynamics in the $K^{-2} 4 a_{1}$ state [35]. Propagation of the nuclear wave packet along the antisymmetric stretching mode leads to considerable additional broadening of the $K^{-2} 4 a_{1}$ contribution in the HS Auger spectrum.

In Fig. 6(c) the wave-packet evolution of the $K^{-2} 2 a_{1}^{-1} 4 a_{1}$ state is shown. Due to the presence of an electron in the $4 a_{1}$ orbital the molecule also experiences antisymmetric motion but the additional vacancy in the $2 a_{1}$ orbital leads to an even faster dissociation, dominated by the symmetric mode and occurring within a few femtoseconds. The evolution of the average $\mathrm{O}-\mathrm{H}$ bond length is shown in Fig. 6(d), demonstrating the different speed of proton dissociation for different DCH states. Note that the dissociative dynamics in the DCH states of water, revealed here in the HS Auger spectra, are susceptible to continue in the following steps of Auger cascade through multiply charged states with few-femtosecond lifetimes, leading eventually to molecular fragmentation [16].

\section{CONCLUSION}

In conclusion, we have demonstrated an experimental indication of ultrafast nuclear dynamics in a doubly-core-ionized and excited water molecule. Although the lifetime of the DCH states is very short, $1.5 \mathrm{fs}$, spectral features of the HS Auger spectrum indicate the presence of ultrafast proton motion that we have identified via $a b$ initio electronic-structure calculations. Theoretical analysis reveals contributions of multiple DCH states created in water molecules irradiated with hard$\mathrm{X}$-ray synchrotron radiation above the $\mathrm{O} K^{-2}$ threshold. Our calculations allow us to disentangle the different dissociation modes involved in the evolution of the molecular geometry in the doubly-core-ionized water molecule. While the dominant $K^{-2}$ DCH state leads mainly to a symmetric nuclear dynamics, other $K^{-2} V$ DCH states undergo even faster dissociative dynamics with a strong antisymmetric character. The results obtained for the gas-phase water are useful for interpretation of dynamic processes occurring in liquid water irradiated with modern hard-X-ray sources. In contrast to resonant spectroscopy, which requires monochromatic radiation at a specific photon energy, HS Auger spectroscopy is pertaining at any photon energy exceeding the double-core-ionization potential and can be equally used at synchrotron and freeelectron-laser sources.

\section{ACKNOWLEDGMENTS}

Experiments were performed on the GALAXIES beamline at SOLEIL Synchrotron, France (Proposals No. 20150921 and No. 99150083). We are grateful to the SOLEIL staff for smoothly running the facility. We thank S. Carniato (Sorbonne Université, France) and E. Shigemasa (UVSOR facility, Japan) for fruitful discussions. T.M. acknowledges financial support from the French Agence Nationale de la Recherche (ANR) through the ATTOMEMUCHO project (ANR-16-CE30-0001).
[1] L. G. M. Pettersson, R. H. Henchman, and A. Nilsson, Chem. Rev. (Washington, DC, U.S.) 116, 7459 (2016).

[2] T. Fransson, Y. Harada, N. Kosugi, N. A. Besley, B. Winter, J. J. Rehr, L. G. M. Pettersson, and A. Nilsson, Chem. Rev. (Washington, DC, U.S.) 116, 7551 (2016).

[3] I. Baccarelli, F. A. Gianturco, E. Scifoni, A. V. Solov'yov, and E. Surdutovich, Eur. Phys. J. D 60, 1 (2010).

[4] K. M. Lange, A. Kothe, and E. F. Aziz, Phys. Chem. Chem. Phys. 14, 5331 (2012).

[5] K. M. Lange and E. F. Aziz, Chem. Soc. Rev. 42, 6840 (2013).
[6] I. Unger, D. Hollas, R. Seidel, S. Thürmer, E. F. Aziz, P. Slavíček, and B. Winter, J. Phys. Chem. B 119, 10750 (2015)

[7] P. Slavíček, N. Kryzhevoi, E. F. Aziz, and B. Winter, J. Phys. Chem. Lett. 7, 234 (2016).

[8] E. Ertan, V. Savchenko, N. Ignatova, V. Vaz da Cruz, R. C. Couto, S. Eckert, M. Fondell, M. Dantz, B. Kennedy, T. Schmitt et al., Phys. Chem. Chem. Phys. 20, 14384 (2018).

[9] D. Céolin, N. V. Kryzhevoi, Ch. Nicolas, W. Pokapanich, S. Choksakulporn, P. Songsiriritthigul, T. Saisopa, Y. 
Rattanachai, Y. Utsumi, J. Palaudoux et al., Phys. Rev. Lett. 119, 263003 (2017).

[10] M. Odelius, H. Ogasawara, D. Nordlund, O. Fuchs, L. Weinhardt, F. Maier, E. Umbach, C. Heske, Y. Zubavichus, M. Grunze et al., Phys. Rev. Lett. 94, 227401 (2005).

[11] A. Nilsson, S. Schreck, F. Perakis, and L. G. M. Pettersson, Adv. Phys.: X 1, 226 (2016).

[12] L. Inhester, G. Groenhof, and H. Grubmüller, J. Chem. Phys. 138, 164304 (2013).

[13] G. Goldsztejn, T. Marchenko, R. Püttner, L. Journel, R. Guillemin, S. Carniato, P. Selles, O. Travnikova, D. Céolin, A. F. Lago et al., Phys. Rev. Lett. 117, 133001 (2016).

[14] M. Žitnik, R. Püttner, G. Goldsztejn, K. Bučar, M. Kavčič, A. Mihelič, T. Marchenko, R. Guillemin, L. Journel, O. Travnikova et al., Phys. Rev. A 93, 021401(R) (2016).

[15] T. Marchenko, G. Goldsztejn, K. Jänkälä, O. Travnikova, L. Journel, R. Guillemin, N. Sisourat, D. Céolin, M. Žitnik, M. Kavčič et al., Phys. Rev. Lett. 119, 133001 (2017).

[16] O. Travnikova, T. Marchenko, G. Goldsztejn, K. Jänkälä, N. Sisourat, S. Carniato, R. Guillemin, L. Journel, D. Céolin, R. Püttner et al., Phys. Rev. Lett. 116, 213001 (2016).

[17] L. Inhester, C. F. Burmeister, G. Groenhof, and H. Grubmüller, J. Chem. Phys. 136, 144304 (2012).

[18] D. Céolin, J. M. Ablett, D. Prieur, T. Moreno, J.-P. Rueff, T. Marchenko, L. Journel, R. Guillemin, B. Pilette, T. Marin et al., J. Electron Spectrosc. Relat. Phenom. 190, 188 (2013).

[19] J.-P. Rueff, J. M. Ablett, D. Céolin, D. Prieur, T. Moreno, V. Balédent, B. Lassalle, J. E. Rault, M. Simon, and A. Shukla J. Synchrotron Radiat. 22, 175 (2015).

[20] M. Mucke, J. H. D. Eland, O. Takahashi, P. Linussond, D. Lebrun, K. Ueda, and R. Feifel, Chem. Phys. Lett. 558, 82 (2013).
[21] G. Goldsztejn, R. Püttner, L. Journel, R. Guillemin, O. Travnikova, B. Cunha de Miranda, I. Ismail, S. Carniato, P. Selles, D. Céolin et al., Phys. Rev. A 96, 012513 (2017).

[22] W. E. Moddeman, T. A. Carlson, M. O. Krause, B. P. Pullen, W. E. Bull, and G. K. Schweitzer, J. Chem. Phys. 55, 2317 (1971).

[23] Eiji Shigemasa, UVSOR Facility, Institute for Molecular Science (private communication).

[24] Cederbaum et al., J. Chem. Phys. 98, 9691 (1993).

[25] J. J. Sakurai, Modern Quantum Mechanics, Revised Edition, edited by San Fu Tuan (Addison-Wesley Publishing Company, Reading, 1994), p. 96.

[26] A. G. Kochur and V. A. Popov, Opt. Spectrosc. 100, 645 (2006).

[27] Stéphane Carniato, Sorbonne Université, Laboratoire de Chimie Physique-Matière et Rayonnement (private communication).

[28] S. Carniato, P. Selles, L. Andric, J. Palaudoux, F. Penent, M. Žitnik, K. Bučar, M. Nakano, Y. Hikosaka, K. Ito et al., J. Chem. Phys. 142, 014308 (2015).

[29] M. U. Kuchiev and S. A. Sheinerman. Usp. Fiz. Nauk 158, 353 (1989).

[30] G. B. Armen, J. Tulkki, T. Åberg, and B. Crasemann, Phys. Rev. A 36, 5606 (1987).

[31] P. Pelicon, I. Čadež, M. Žitnik, Ž. Šmit, S. Dolenc, A. Mühleisen, and R. I. Hall, Phys. Rev. A 62, 022704 (2000).

[32] S. Carniato, J. Chem. Phys. 126, 224307 (2007).

[33] O. Takahashi, N. Kunitake, and S. Takaki, J. Phys. B: At., Mol. Opt. Phys. 48, 204001 (2015).

[34] I. Hjelte, M. N. Piancastelli, R. F. Fink, O. Björneholm, M. Bässler, R. Feifel, A. Giertz, H. Wang, K. Wiesner, A. Ausmees et al., Chem. Phys. Lett. 334, 151 (2001).

[35] S. Carniato, P. Selles, L. Andric, J. Palaudoux, F. Penent, M. Žitnik, K. Bučar, M. Nakano, Y. Hikosaka, K. Ito et al., J. Chem. Phys. 142, 014307 (2015). 